\title{
Photoredox-Catalyzed Ketyl-Olefin Coupling for the Synthesis of Substituted Chromanols
}

\author{
Eleonora Fava, ${ }^{\dagger, \dagger}$ Masaki Nakajima, ${ }^{\dagger}$ Anh L. P. Nguyen, ${ }^{\dagger}$ and Magnus Rueping ${ }^{*}, \dagger, \ddagger$ \\ ${ }^{\dagger}$ RWTH Aachen University, Institute of Organic Chemistry, Landoltweg 1, 52074, Aachen, Germany \\ ${ }^{\ddagger}$ King Abdullah University of Science and Technology (KAUST), KAUST Catalysis Center (KCC), Thuwal, 23955-6900, Saudi \\ Arabia
}

Supporting Information

ABSTRACT: A visible light photoredox-catalyzed aldehyde olefin cyclization is reported. The method represents a formal hydroacylation of alkenes and alkynes and provides chromanol derivatives in good yields. The protocol takes advantage of the double role played by trialkylamines $\left(\mathrm{NR}_{3}\right)$ which act as (i) electron donors for reducing the catalyst and (ii) proton donors to activate the substrate via a proton-coupled electron transfer.

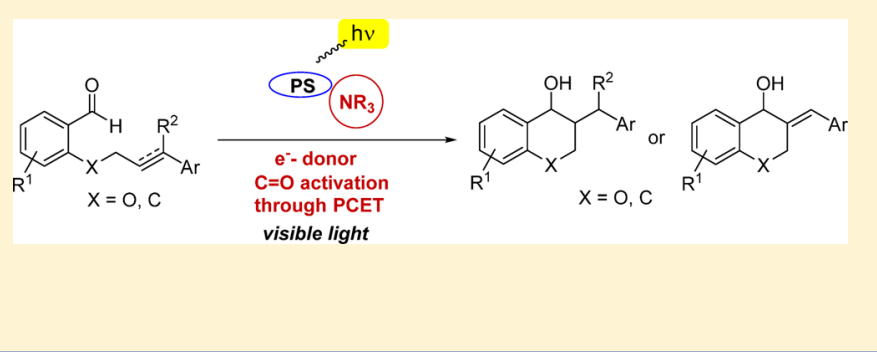

Knowles and co-workers reported a catalytic protocol in which ketones are successfully reduced to ketyl radicals by the concerted transfer of both a proton and an electron. ${ }^{11 \mathrm{~b}}$ This phenomenon is named proton-coupled electron transfer (PCET) ${ }^{15}$

In an attempt to demonstrate the active role of proton donors in the reduction step, Knowles and co-workers also reported an asymmetric aza-pinacol cyclization of ketones and hydrazones where the binding effect of the chiral phosphoric acids led to the formation of enantioenriched vicinal amino alcohols. $^{11 \mathrm{c}}$

Recently, our group described the photoredox-catalyzed pinacol coupling of aldehydes, ketones, and aldimines through hydrogen-bonding activation. ${ }^{12 a}$ With this work, we disclose the possibility of generating a long-lived ketyl radical using tertiary amines as the electron and proton source, replacing the combination of Hantzsch ester and diphenyl phosphate (DPP).

Based on our previous work, ${ }^{12 a}$ we envisioned the possibility of performing the ketyl radical addition on unsaturated bonds triggered by visible light, which would initiate the singleelectron reduction from a photoexcited polypyridyl iridium complex to a carbonyl group (aldehyde or ketone). We postulated that the so-generated radical intermediate would be able to undergo subsequent intramolecular addition to a pendant alkene or alkyne moiety (Scheme 1 ).

Herein, we report the realization of this activation mode in the context of a catalytic intramolecular reductive coupling of aldehydes with alkenes and alkynes. This transformation allows the synthesis of substituted 3-benzylchroman-4-ols (5), containing a chroman unit, that exhibit anti picornavirus

Special Issue: Photocatalysis

Received: April 30, 2016

Published: July 21, 2016 
Scheme 1. Photoredox-Catalyzed Reduction of Aldehydes through PCET

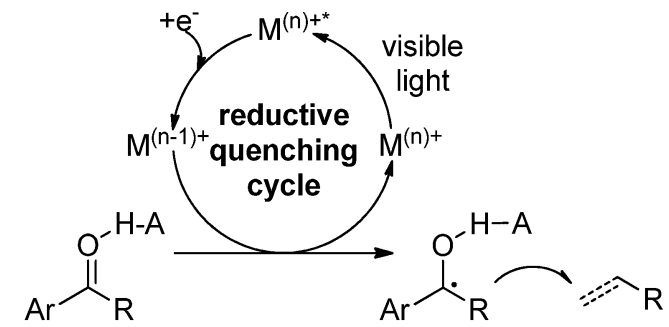

$\mathrm{R}=\mathrm{H}$ or $\mathrm{Me}$

Ketyl radical

activity, commonly responsible for the upper respiratory tract infections in humans. ${ }^{16}$

Remarkably, we observed that tertiary amines not only provided the desired products in higher yields but also proved to be crucial for the reaction to proceed.

We began our studies by evaluating conditions for the cyclization of 2-(cinnamyloxy)benzaldehyde (1a) to chromanol $\mathbf{2 a}$ in the presence of $\operatorname{Ir}(\mathrm{ppy})_{2}(\mathrm{dtb}-\mathrm{bpy}) \mathrm{PF}_{6}$ (PC 3, $\left.1 \mathrm{~mol} \%\right)$, trialkylamines $4 \mathbf{a}-\mathbf{c}$ as reductive quencher (RQ), and irradiation with a blue LED light source $\left(11 \mathrm{~W}, \lambda_{\max }=450\right.$ $\mathrm{nm}$ ). As shown in Table S1 (Supporting Information), the reaction proceeded with good yield in the presence of 2.5 equiv of tributylamine ( $4 \mathbf{b}$, entry 5 ). Solvent evaluation confirmed acetonitrile to be the best solvent, whereas aprotic (entries 1 and 2) and protic (entry 3) polar solvents were shown to be unsuccessful as reaction media. Further screening of electron donors in acetonitrile confirmed Hünig base (4c) to be the best among the trialkylamines tested, providing the desired product in $80 \%$ yield (entry 6 ). As previously mentioned, the use of Hantzsch esters $\mathbf{5 a}$ and $\mathbf{5 b}$ alone or in combination with $\mathbf{1 0} \mathrm{mol}$ $\%$ of DPP did not lead to the expected product (entries 7-10). A series of control experiments demonstrated that the reaction did not take place in the absence of light, catalyst, or 3. With the optimized conditions in hand (acetonitrile, rt, $1 \mathrm{~mol} \%$ PC 3 ), we began to evaluate a variety of substrates (Table 1 ).

As shown in Table 1, the reaction tolerates a wide variety of substituents, confirming the ability of the ketyl radical to couple to the olefin in the presence of both electron-withdrawing and -donating groups on the aromatic aldehyde moiety.

Following the development of a successful formal hydroacylation we investigated the reaction in which the olefin was replaced by an alkyne group (Table 2). Also in this case, the reaction gave the expected products (2o and $2 p$ ) in good yields.

Regarding the reaction mechanism, we propose the following: Irradiation with visible light results in the formation of the reductive species $\mathrm{Ir}^{2+}$. Hünig base $\left(i-\mathrm{Pr}_{2} \mathrm{NEt}\right)$ acts as a sacrificial electron donor; therefore, the single-electron oxidation weakens the adjacent $\mathrm{C}-\mathrm{H}$ bond ${ }^{17}$ and the $1,2-\mathrm{H}$ shift becomes energetically favored. The following deprotonation through the tertiary amine provides the ammonium derivative, which is now able to engage in a proton-coupled electron transfer with the substrate, lowering its potential for $\mathrm{Ir}^{2+}$ reduction and generation of a neutral ketyl intermediate. Alternatively, the ammonium radical could also activate the carbonyl functionality. The ketyl radical is able to add to the unsaturated bond, forming the chroman ring and a benzyl radical which upon hydrogen atom transfer leads to the final product (Scheme 2).
Table 1. Scope of the Reaction ${ }^{a-c}$
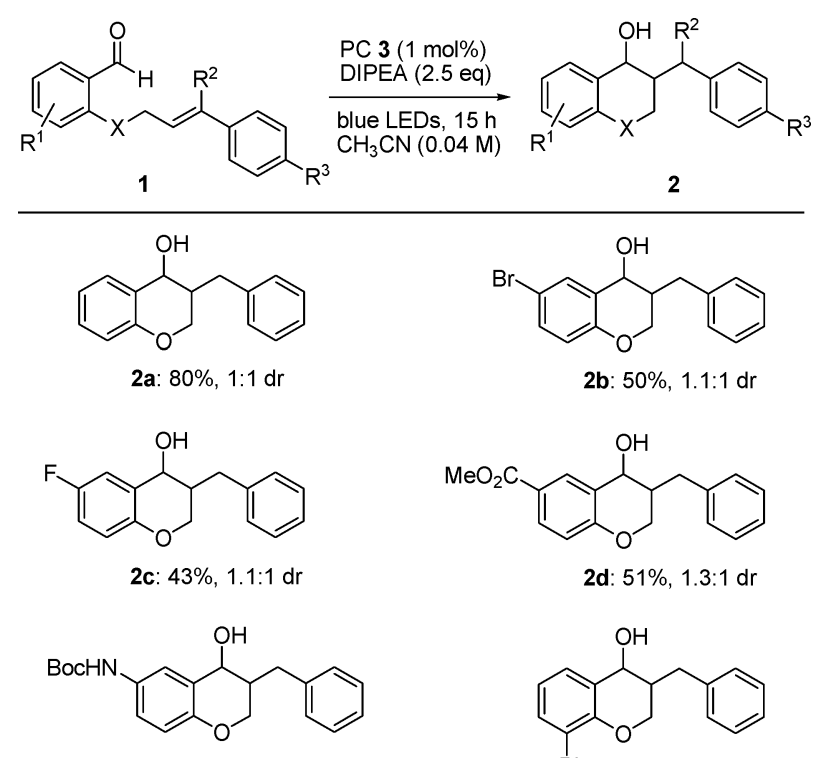

2e: $44 \%, 1.6: 1 \mathrm{dr}$
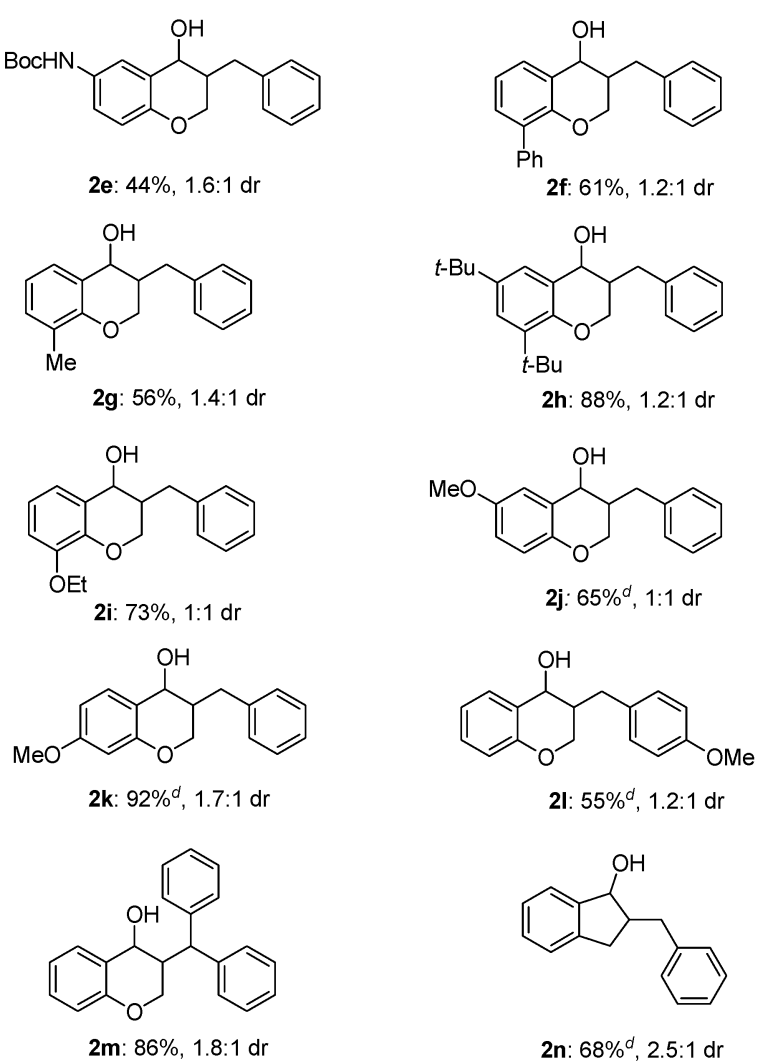

2n: $68 \%^{d}, 2.5: 1 \mathrm{dr}$

${ }^{a}$ All reactions of $1(0.12 \mathrm{mmol})$ with DIPEA $(0.3 \mathrm{mmol})$ were carried out in the presence of PC $3(1 \mathrm{~mol} \%)$ in acetonitrile $(3.0 \mathrm{~mL})$ under irradiation with blue LEDs $(11 \mathrm{~W}, 450 \mathrm{~nm})$ for $15 \mathrm{~h}$ at $25{ }^{\circ} \mathrm{C}$. ${ }^{b}$ Isolated yields are reported. ${ }^{c} \mathrm{The} \mathrm{dr}$ is given for anti/syn ratio. ${ }^{d}$ Reaction performed using $2.5 \mathrm{~mol} \%$ of photocatalyst.

\section{CONCLUSIONS}

In conclusion, we report a ketyl-olefin coupling for the preparation of substituted 3-benzylchroman-4-ols, promoted by visible light photoredox catalysis. Importantly, we uncovered trialkylamines as a cheap and readily available alternative to the previously reported electron-proton donor system consisting of Hantzsch ester/Brønsted acid. By employing the trialkylamine/photocatalyst system, we have been able to develop an efficient intramolecular ketyl-olefin and ketyl-alkyne coupling employing readily prepared substrates. The formal hydroacylation protocol provides the chromanol derivatives in good yield under mild reaction conditions and with low catalyst 
Table 2. Scope of the Intramolecular Ketyl-Alkyne Cyclization $^{a}$<smiles>O=Cc1ccccc1OCC#CBr</smiles>

1

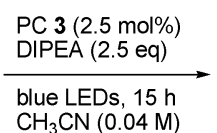

$\mathrm{CH}_{3} \mathrm{CN}(0.04 \mathrm{M})$

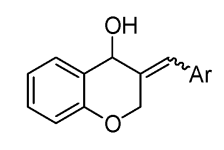

2

\begin{tabular}{clcc} 
entry & \multicolumn{1}{c}{ product } & yield $^{b}(\%)$ & $E / Z$ \\
1 & $\mathrm{Ar}=m-\mathrm{MePh}(\mathbf{2 o})$ & 57 & $1: 1$ \\
2 & $\mathrm{Ar}=p-\mathrm{OMePh}(\mathbf{2 p})$ & 59 & $1: 1$
\end{tabular}

${ }^{a}$ All reactions of $1(0.12 \mathrm{mmol})$ with DIPEA $(0.3 \mathrm{mmol})$ were carried out in the presence of PC $3(2.5 \mathrm{~mol} \%)$ in acetonitrile $(3.0 \mathrm{~mL})$ under irradiation with blue LEDs $(11 \mathrm{~W}, 450 \mathrm{~nm})$ for $18 \mathrm{~h}$ at $25{ }^{\circ} \mathrm{C}$. ${ }^{b}$ Isolated yields are reported.

loadings. Efforts are currently underway to expand this concept to further transformations.

\section{EXPERIMENTAL SECTION}

General Information. All reactions were performed with ovendried glassware and under an inert atmosphere (argon) unless otherwise stated. Acetonitrile was distilled from calcium hydride and stored over $4 \AA$ molecular sieves under nitrogen/argon atmosphere. Tetrahydrofuran was distilled from Solvona/benzophenone. Pentane was distilled in a standard distillation apparatus. Other solvents were used as purchased unless otherwise stated. Commercial reagents were used as purchased without further purification. Organic solutions were concentrated under reduced pressure on a rotary evaporator. Chromatographic purification of products was carried out using silica gel (230-400 mesh). Thin-layer chromatography was carried out using aluminum plates coated with silica (230-400 mesh) which were visualized under UV light $(254 \mathrm{~nm})$ or by staining with aqueous potassium permanganate solutions or vanillin alcoholic solution. ${ }^{1} \mathrm{H}$ NMR spectra were recorded in deuterated solvents on spectrometers at 300,400 , or $600 \mathrm{MHz}$ with residual protic solvent as the internal standard. ${ }^{13} \mathrm{C}$ NMR spectra were recorded in deuterated solvents on spectrometers at 75,101 , or $151 \mathrm{MHz}$ with the central peak of the deuterated solvent as the internal standard. Chemical shifts $(\delta)$ are given in parts per million (ppm), and coupling constants $(J)$ are given in hertz $(\mathrm{Hz})$ rounded to the nearest $0.1 \mathrm{~Hz}$. The ${ }^{1} \mathrm{H}$ NMR spectra are reported as $\delta$ downfield from tetramethylsilane (multiplicity, coupling constant $J$, number of protons). The ${ }^{13} \mathrm{C}$ NMR spectra are reported as $\delta$. For the F-containing compound $(\mathbf{2 c})$, a simultaneously proton and fluorine decoupled ${ }^{13} \mathrm{C}$ NMR spectrum was recorded. Assignments are aided by the use of DEPT-135, COSY and HMQC spectra where necessary. IR spectra were recorded on a FTIR spectrometer. Low resolution mass spectra (EI/CI) were recorded on a mass spectrometer with (EI) or (CI) detectors. High-resolution mass spectrometry (HRMS) was performed by EI using a double-focusing mass spectrometer and by ESI using a hybrid ion-trap mass spectrometer. Melting points were recorded at ambient pressure and are uncorrected.

General Procedure for the Synthesis of Chromanols. A Schlenk tube was charged with aldehyde 1 (1 equiv), catalyst PC 3 (1 or $2.5 \mathrm{~mol} \%)$, and degassed DIPEA (2.5 equiv). It was capped, evacuated, and backfilled with argon. Subsequently, degassed acetonitrile $(3 \mathrm{~mL})$ was added via syringe. The vial was placed in a $100 \mathrm{~mL}$ beaker containing a blue LED strip glued on the inner wall, and the reaction mixture was stirred for $15 \mathrm{~h}$. After this time, the solvent was evaporated, and the reaction mixture was purified on silica gel (gradient pentane/EtOAc 95:5).

3-Benzylchroman-4-ol (2a). ${ }^{18}$ The title compound was synthesized according to the general procedure employing $1 \mathrm{a}(0.12 \mathrm{mmol}, 28.6$ $\mathrm{mg}), 1 \mathrm{~mol} \%(1.1 \mathrm{mg})$ of PC 3 , and 2.5 equiv of DIPEA $(38.8 \mathrm{mg})$. The product was purified by flash column chromatography: yield $80 \%$ (23 mg); anti/syn 1:1; ${ }^{1} \mathrm{H}$ NMR $\left(600 \mathrm{MHz}, \mathrm{CDCl}_{3}\right) \delta 7.34-7.18(\mathrm{~m}$, $14 \mathrm{H}), 6.96(\mathrm{td}, J=7.5,0.9 \mathrm{~Hz}, 1 \mathrm{H}), 6.90(\mathrm{dd}, J=10.6,4.2 \mathrm{~Hz}, 2 \mathrm{H})$, $6.85(\mathrm{~d}, J=8.5 \mathrm{~Hz}, 1 \mathrm{H}), 4.51(\mathrm{dd}, J=9.4,3.1 \mathrm{~Hz}, 2 \mathrm{H}), 4.23(\mathrm{dd}, J=$ $11.1,2.6 \mathrm{~Hz}, 1 \mathrm{H}), 4.14-4.05(\mathrm{~m}, 2 \mathrm{H}), 3.98(\mathrm{dd}, J=11.1,4.2 \mathrm{~Hz}, 1 \mathrm{H})$, $2.89(\mathrm{dd}, J=13.8,8.4 \mathrm{~Hz}, 1 \mathrm{H}), 2.75-2.64(\mathrm{~m}, 2 \mathrm{H}), 2.54(\mathrm{dd}, J=13.8$, $9.3 \mathrm{~Hz}, 1 \mathrm{H}), 2.40-2.29(\mathrm{~m}, 1 \mathrm{H}), 2.28-2.17(\mathrm{~m}, 1 \mathrm{H}), 1.92(\mathrm{bs}, 1 \mathrm{H})$, 1.74 (bs, $1 \mathrm{H}) ;{ }^{13} \mathrm{C}$ NMR $\left(151 \mathrm{MHz}, \mathrm{CDCl}_{3}\right) \delta 154.3,154.2,139.2$, 139.1, 130.2, 130.1, 129.9, 129.8, 129.1 (2C), 128.6, 128.5, 126.3 (2C), $124.1,123.2,120.9,120.5,116.9$ (2C), 67.6, 64.9 (2C), 64.6, 41.5, 40.0, 34.6, 32.8; FT-IR $\nu_{\max }$ (ATR) $\mathrm{cm}^{-1} 3372,2922,1582,1485,1450$, $1302,1264,1222,1119,1074,1039,909,748,698 ; \mathrm{m} / z$ (EI) 240 $\left([\mathrm{M}]^{+}, 100\right), 91\left(\left[\mathrm{PhCH}_{2}\right]^{+}, 28\right)$; HRMS (ESI) calcd for $\left[\mathrm{C}_{16} \mathrm{H}_{16} \mathrm{O}_{2}+\right.$ $\mathrm{Na}]^{+}$263.10425, found 263.10419 .

3-Benzyl-6-bromochroman-4-ol (2b). The title compound was synthesized according to the general procedure employing $\mathbf{1 b}(0.12$ mmol, $38.1 \mathrm{mg}), 2.5 \mathrm{~mol} \%(2.74 \mathrm{mg})$ of PC 3 , and 2.5 equiv of DIPEA $(38.8 \mathrm{mg})$. The product was purified by flash column chromatography: yield $50 \%$ (19.1 mg); anti/syn 1.1:1; ${ }^{1} \mathrm{H}$ NMR (400 $\left.\mathrm{MHz}, \mathrm{CD}_{3} \mathrm{OD}\right) \delta 7.45(\mathrm{~d}, J=2.4 \mathrm{~Hz}, 1 \mathrm{H}, s y n), 7.34(\mathrm{~d}, J=3.1 \mathrm{~Hz}, 1.1$ $\times 1 \mathrm{H}$, anti), $7.31-7.13(\mathrm{~m}, 12.6 \mathrm{H}), 6.73-6.65(\mathrm{~m}, 2.1 \mathrm{H}), 4.47(\mathrm{~d}, J=$ $3.4 \mathrm{~Hz}, 1 \mathrm{H}$, syn), $4.36(\mathrm{~d}, J=5.0 \mathrm{~Hz}, 1.1 \times 1 \mathrm{H}$, anti), $4.13(\mathrm{dd}, J=$ $11.2,2.8 \mathrm{~Hz}, 1.1 \times 1 \mathrm{H}$, anti) $, 4.03-3.94(\mathrm{~m}, 2 \mathrm{H}$, syn $), 3.89(\mathrm{dd}, J=$

Scheme 2. Plausible Reaction Mechanism

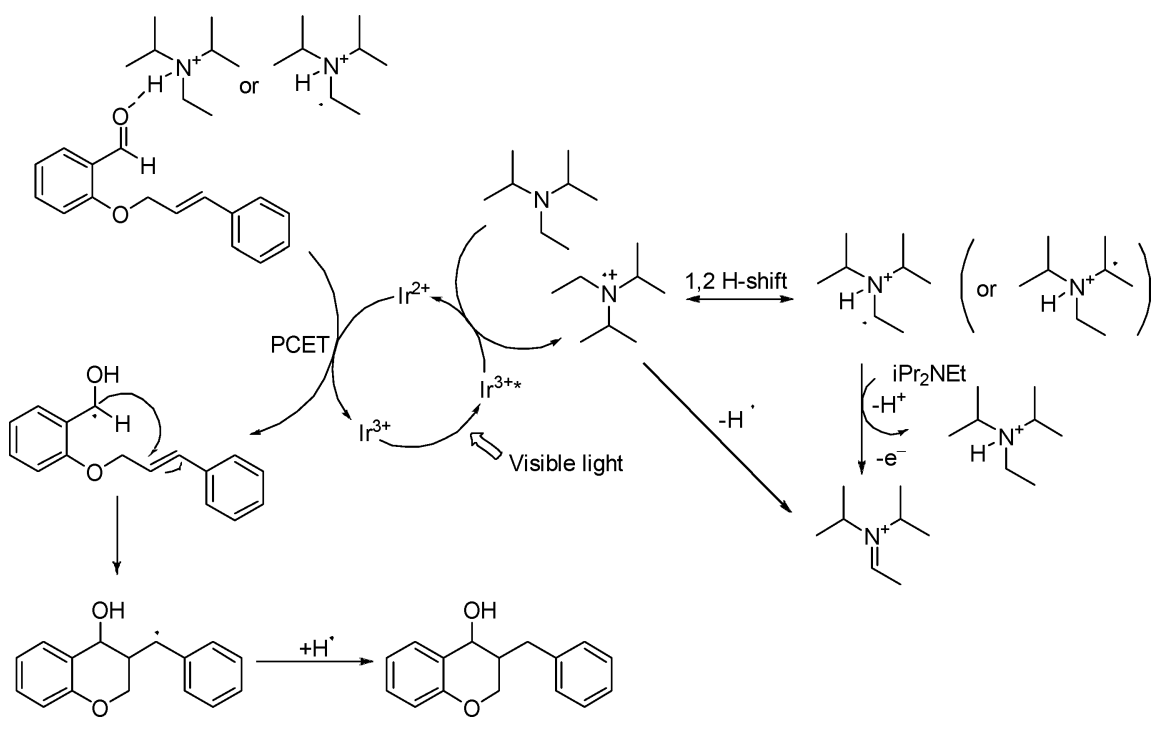


11.1, $5.5 \mathrm{~Hz}, 1.1 \times 1 \mathrm{H}$, anti), $2.87(\mathrm{dd}, J=13.6,7.1 \mathrm{~Hz}, 1 \mathrm{H}$, syn), 2.76 (dd, $J=13.8,6.1 \mathrm{~Hz}, 1 \mathrm{H}$, syn), $2.55(\mathrm{dd}, J=13.6,8.3 \mathrm{~Hz}, 1.1 \times 1 \mathrm{H}$, anti), $2.45(\mathrm{dd}, J=13.7,9.3 \mathrm{~Hz}, 1.1 \times 1 \mathrm{H}$, anti), $2.25-2.17(\mathrm{~m}, 1 \mathrm{H}$, syn), 2.16-2.08 (m, $1.1 \times 1 \mathrm{H}$, anti); ${ }^{13} \mathrm{C}$ NMR $\left(101 \mathrm{MHz}, \mathrm{CD}_{3} \mathrm{OD}\right) \delta$ 153.5 (2C), 139.4, 139.2, 132.4 (2C), 131.60 (2C), 128.8, 128.7, 128.1 (2C), 127.0, 126.1, 126.0, 125.8, 118.1 (2C), 111.9, 111.5, 66.4, 65.0, 64.8, 64.2, 41.5, 40.2, 34.2, 32.1; FT-IR $\nu_{\max }$ (ATR) $\mathrm{cm}^{-1} 2926,1479$, $1407,1260,1224,1191,1085,1055,1021,819,748,699 ; \mathrm{m} / z$ (EI) $319\left([\mathrm{M}]^{+}, 9\right)$, $91\left(\left[\mathrm{PhCH}_{2}\right]^{+}, 100\right)$; HRMS (EI) calcd for $\left[\mathrm{C}_{16} \mathrm{H}_{15} \mathrm{O}_{2}^{79} \mathrm{Br}\right]^{+}$318.02499, found 318.02492.

3-Benzyl-6-fluorochroman-4-ol (2c). The title compound was synthesized according to the general procedure employing 1c $(0.12$ mmol, $30.8 \mathrm{mg}), 1 \mathrm{~mol} \%(1.1 \mathrm{mg})$ of PC 3 and 2.5 equiv of DIPEA $(38.8 \mathrm{mg})$. The product was purified by flash column chromatography: yield $43 \%$ (13.2 mg); mp $=102-104{ }^{\circ} \mathrm{C}$; anti $/$ syn $1.1: 1 ;{ }^{1} \mathrm{H}$ NMR $(600$ $\left.\mathrm{MHz}, \mathrm{CD}_{3} \mathrm{OD}\right) \delta 7.31-7.23(\mathrm{~m}, 6.5 \mathrm{H}), 7.21-7.15(\mathrm{~m}, 4.3 \mathrm{H}), 7.06$ $(\mathrm{dd}, J=9.0,3.1 \mathrm{~Hz}, 1.1 \times 1 \mathrm{H}$, anti), $6.96(\mathrm{dd}, J=8.9,3.1 \mathrm{~Hz}, 1 \mathrm{H}$, syn $)$, $6.89(\mathrm{dtd}, J=11.6,8.5,3.1 \mathrm{~Hz}, 2 \mathrm{H}), 6.78-6.71(\mathrm{~m}, 2 \mathrm{H}), 4.48(\mathrm{~d}, J=$ $3.5 \mathrm{~Hz}, 1 \mathrm{H}, \operatorname{syn}), 4.37(\mathrm{~d}, J=5.1 \mathrm{~Hz}, 1.1 \times 1 \mathrm{H}$, anti), $4.12(\mathrm{dd}, J=$ 11.1, $2.8 \mathrm{~Hz}, 1.1 \times 1 \mathrm{H}$, anti) $, 4.01-3.94(\mathrm{~m}, 2 \mathrm{H}$, syn $), 3.87(\mathrm{dd}, J=$ 11.1, $5.6 \mathrm{~Hz}, 1.1 \times 1 \mathrm{H}$, anti), $2.88(\mathrm{dd}, J=13.6,7.2 \mathrm{~Hz}, 1 \mathrm{H}$, syn), 2.79 (dd, $J=13.8,6.0 \mathrm{~Hz}, 1.1 \times 1 \mathrm{H}$, anti), $2.56(\mathrm{dd}, J=13.6,8.3 \mathrm{~Hz}, 1 \mathrm{H}$, syn), $2.46(\mathrm{dd}, J=13.8,9.4 \mathrm{~Hz}, 1.1 \times 1 \mathrm{H}$, anti), 2.24-2.17 (m, $1 \mathrm{H}$, syn), $2.16-2.11\left(\mathrm{~m}, 1.1 \times 1 \mathrm{H}\right.$, anti); ${ }^{19} \mathrm{~F} \mathrm{NMR}\left(564 \mathrm{MHz}, \mathrm{CD}_{3} \mathrm{OD}\right) \delta$ $-125.8,-126.4 ;{ }^{13} \mathrm{C}$ NMR $\left(101 \mathrm{MHz}, \mathrm{CD}_{3} \mathrm{OD}\right) \delta 156.9,156.6,150.4$, $150.3,139.5,139.2,128.8,128.7,128.1,128.0,125.9$ (2C), 125.8, 125.0, 117.2, 117.1, 115.5, 115.4, 115.3 (2C), 66.8, 65.0, 64.7, 64.4, 41.6, 40.3, 34.2, 32.1; FT-IR $\nu_{\max }$ (ATR) $\mathrm{cm}^{-1} 3370,2926,2493,1488$, $1436,1251,1201,1027,736,698 ; m / z$ (EI) $258\left([\mathrm{M}]^{+}, 48\right), 91$ $\left(\left[\mathrm{PhCH}_{2}\right]^{+}, 100\right)$; HRMS (EI) calcd for $\left[\mathrm{C}_{16} \mathrm{H}_{15} \mathrm{O}_{2} \mathrm{~F}\right]^{+}$258.10506, found 258.10537 .

Methyl 3-Benzyl-4-hydroxychroman-6-carboxylate (2d). The title compound was synthesized according to the general procedure employing 1d $(0.12 \mathrm{mmol}, 35.6 \mathrm{mg}), 1 \mathrm{~mol} \%(1.1 \mathrm{mg})$ of PC 3, and 2.5 equiv of DIPEA $(38.8 \mathrm{mg})$. The product was purified by flash column chromatography: yield $51 \%(18.2 \mathrm{mg})$; anti/syn $1.3: 1 ;{ }^{1} \mathrm{H}$ NMR $\left(400 \mathrm{MHz}, \mathrm{CD}_{3} \mathrm{OD}\right) \delta 8.05(\mathrm{~d}, J=2.1 \mathrm{~Hz}, 1.3 \times 1 \mathrm{H}$, anti), 7.92 (d, $J=2.2 \mathrm{~Hz}, 1 \mathrm{H}$, syn), 7.80 (ddd, $J=14.4,8.6,2.2 \mathrm{~Hz}, 2.3 \mathrm{H}$ ), 7.32$7.11(\mathrm{~m}, 11.5 \mathrm{H}), 6.81(\mathrm{dd}, J=14.4,8.6 \mathrm{~Hz}, 2.3 \mathrm{H}), 4.52(\mathrm{~d}, J=3.2 \mathrm{~Hz}$, $1 \mathrm{H}$, syn), $4.42(\mathrm{~d}, J=4.9 \mathrm{~Hz}, 1.3 \times 1 \mathrm{H}$, anti), $4.21(\mathrm{dd}, J=11.2,2.9$ $\mathrm{Hz}, 1.3 \times 1 \mathrm{H}$, anti), $4.11-3.99(\mathrm{~m}, 2 \mathrm{H}$, syn $), 3.96(\mathrm{dd}, J=11.1,5.3 \mathrm{~Hz}$, $1.3 \times 1 \mathrm{H}$, anti), $3.84(\mathrm{~s}, 1.3 \times 3 \mathrm{H}$, anti $), 3.81(\mathrm{~s}, 3 \mathrm{H}$, syn $), 2.88(\mathrm{dd}, J=$ 13.6, 7.1 Hz, 1H, syn), 2.75 (dd, $J=13.7,6.1 \mathrm{~Hz}, 1.3 \times 1 \mathrm{H}$, anti), 2.56 (dd, $J=13.6,8.3 \mathrm{~Hz}, 1 \mathrm{H}$, syn), $2.43(\mathrm{dd}, J=13.7,9.3 \mathrm{~Hz}, 1.3 \times 1 \mathrm{H}$, anti), 2.26-2.13 (m, 2.3H); ${ }^{13} \mathrm{C}$ NMR (101 MHz, $\left.\mathrm{CD}_{3} \mathrm{OD}\right) \delta 167.0$ (2C), $158.6(2 \mathrm{C}), 139.4,139.1,132.3,132.2,130.4,130.3,128.8$, $128.7,128.1$ (2C), 126.0, 125.9, 124.8, 123.9, 122.1, 121.7, 116.3 (2C), 66.4, 65.4, 65.2, 64.2, 51.0, 50.9, 41.4, 40.2, 34.2, 32.2; FT-IR $\nu_{\max }($ ATR $) \mathrm{cm}^{-1} 2949,1711,1611,1496,1436,1253,1192,1125$, $1012,767,700 ; \mathrm{m} / z(\mathrm{EI}) 298\left([\mathrm{M}]^{+}, 12\right), 91\left(\left[\mathrm{PhCH}_{2}\right]^{+}, 40\right) ; \mathrm{HRMS}$ (EI) calcd for $\left[\mathrm{C}_{18} \mathrm{H}_{18} \mathrm{O}_{4}\right]^{+} 298.11996$, found 298.12009.

tert-Butyl (3-Benzyl-4-hydroxychroman-6-yl)carbamate (2e). The title compound was synthesized according to the general procedure employing 1e $(0.12 \mathrm{mmol}, 42.4 \mathrm{mg}), 1 \mathrm{~mol} \%(1.1 \mathrm{mg})$ of PC 3, and 2.5 equiv of DIPEA $(38.8 \mathrm{mg})$. The product was purified by flash column chromatography: yield $44 \%(18.7 \mathrm{mg})$; anti/syn $1.6: 1 ;{ }^{1} \mathrm{H}$ NMR $\left(600 \mathrm{MHz}, \mathrm{CDCl}_{3}\right) \delta 7.49-7.15(\mathrm{~m}, 15.6 \mathrm{H}), 7.08(\mathrm{dd}, J=8.8$, $2.6 \mathrm{~Hz}, 1.6 \times 1 \mathrm{H}$, anti), $6.99(\mathrm{dd}, J=8.8,2.6 \mathrm{~Hz}, 1 \mathrm{H}, \mathrm{syn}), 6.80(\mathrm{~d}, J=$ $8.8 \mathrm{~Hz}, 1.6 \times 1 \mathrm{H}$, anti), $6.76(\mathrm{~d}, J=8.8 \mathrm{~Hz}, 1 \mathrm{H}, \mathrm{syn}), 6.45-6.24(\mathrm{~m}$, $2.6 \mathrm{H}), 4.52-4.41(\mathrm{~m}, 2.6 \mathrm{H}), 4.19(\mathrm{dd}, J=11.0,1.9 \mathrm{~Hz}, 1.6 \times 1 \mathrm{H}$, anti), $4.12-4.01(\mathrm{~m}, 2 \mathrm{H}$, syn), $3.92(\mathrm{dd}, J=11.0,3.9 \mathrm{~Hz}, 1.6 \times 1 \mathrm{H}$, anti), $2.86(\mathrm{dd}, J=13.5,8.6 \mathrm{~Hz}, 1 \mathrm{H}$, syn $), 2.73-2.61(\mathrm{~m}, 2.6 \mathrm{H}), 2.51$ (dd, $J=13.7,9.5 \mathrm{~Hz}, 1.6 \times 1 \mathrm{H}$, anti), $2.31-2.26(\mathrm{~m}, 1 \mathrm{H}$, syn), $2.21-$ $2.17(\mathrm{~m}, 1.6 \times 1 \mathrm{H}$, anti), 1.91-1.62 (overlapping of two bs, $2.6 \mathrm{H}$ ) $1.51\left(\mathrm{~s}, 1.6 \times 9 \mathrm{H}\right.$, anti), $1.48\left(\mathrm{~s}, 9 \mathrm{H}\right.$, syn); ${ }^{13} \mathrm{C} \mathrm{NMR}(151 \mathrm{MHz}$, $\left.\mathrm{CDCl}_{3}\right) \delta 153.2,153.1,150.3,150.2,139.2,139.1,131.4,131.3,131.0$ (2C), 129.1 (2C), 128.5 (2C), 126.3 (2C), 124.4, 123.5, 121.2, 120.7, 117.2, 117.1, 80.4 (2C), 67.6, 65.1, 64.8, 64.6, 41.3, 40.0, 34.6, 32.7, 28.4, 28.3; FT-IR $\nu_{\max }$ (ATR) $\mathrm{cm}^{-1}: 3350,2981,2932,1696,1499$, $1454,1367,1309,1241,1218,1163,1023,822,733,699 ; \mathrm{m} / z$ (EI)
$298\left(\left[\mathrm{M}-\left(\mathrm{CH}_{3}\right)_{3} \mathrm{C}\right]^{+}, 100\right), 91\left(\left[\mathrm{PhCH}_{2}\right]^{+}, 67\right)$; HRMS (EI) calcd for $\left[\mathrm{C}_{16} \mathrm{H}_{16} \mathrm{O}_{2} \mathrm{~N}_{1}\right]^{+}\left([\mathrm{M}-\mathrm{Boc}]^{+}\right)$254.11756, found 254.11706.

3-Benzyl-8-phenylchroman-4-ol (2f). The title compound was synthesized according to the general procedure employing if $(0.12$ mmol, $37.7 \mathrm{mg}$ ), $1 \mathrm{~mol} \%$ (1.1 mg) of PC 3, and 2.5 equiv of DIPEA $(38.8 \mathrm{mg})$. The product was purified by flash column chromatography: yield $61 \%$ (23 mg); anti/syn 1.2:1; ${ }^{1} \mathrm{H}$ NMR (600 MHz, $\left.\mathrm{CD}_{3} \mathrm{OD}\right)$ S7.48-7.44 (m, $1.2 \times 1 \mathrm{H}$, anti), 7.44-7.40 (m, $1 \mathrm{H}$, syn $), 7.37-7.05$ $(\mathrm{m}, 24.2 \mathrm{H}), 7.00-6.94(\mathrm{~m}, 1.2 \times 1 \mathrm{H}$, anti $), 6.92-6.86(\mathrm{~m}, 1 \mathrm{H}, \mathrm{syn})$, $4.49(\mathrm{~d}, J=2.7 \mathrm{~Hz}, 1 \mathrm{H}$, syn $), 4.44(\mathrm{~d}, J=4.1 \mathrm{~Hz}, 1.2 \times 1 \mathrm{H}$, anti), 4.15 (dd, $J=11.0,2.6 \mathrm{~Hz}, 1.2 \times 1 \mathrm{H}$, anti), $4.00(\mathrm{t}, J=10.9 \mathrm{~Hz}, 1 \mathrm{H}$, syn $)$, 3.92 (dd, $J=10.6,3.5 \mathrm{~Hz}, 1 \mathrm{H}$, syn), 3.86 (dd, $J=11.0,4.5 \mathrm{~Hz}, 1.2 \times$ $1 \mathrm{H}$, anti), 2.85 (dd, $J=13.5,7.5 \mathrm{~Hz}, 1 \mathrm{H}$, syn), 2.70 (dd, $J=13.7,6.4$ $\mathrm{Hz}, 1.2 \times 1 \mathrm{H}$, anti), $2.55(\mathrm{dt}, J=18.0,9.0 \mathrm{~Hz}, 1 \mathrm{H}$, syn $), 2.51-2.44(\mathrm{~m}$, $1.2 \times 1 \mathrm{H}$, anti), $2.25-2.18(\mathrm{~m}, 1 \mathrm{H}$, syn $), 2.17-2.12(\mathrm{~m}, 1.2 \times 1 \mathrm{H}$, anti); ${ }^{13} \mathrm{C}$ NMR (151 MHz, CD $\left.\mathrm{OD}\right) \delta 151.1$ (2C), 139.6, 139.4, 138.5, 138.4, 130.2, 130.1, 129.8 (2C), 129.7 (2C), 129.2 (2C), 128.8, $128.7,128.1$ (2C), 127.5 (2C), 126.4 (2C), 125.9, 125.8, 125.1, 123.9, 120.2, 119.9, 67.1, 64.8, 64.6, 64.5, 41.5, 40.3, 34.3, 32.5; FT-IR $\nu_{\max }($ ATR $) \mathrm{cm}^{-1} 1594,1496,1469,1430,1219,1017,754,697 ; \mathrm{m} / \mathrm{z}$ (EI) $316\left([\mathrm{M}]^{+}, 63 \%\right), 91\left(\left[\mathrm{PhCH}_{2}\right]^{+}, 27 \%\right)$; HRMS (ESI) calcd for $\left[\mathrm{C}_{22} \mathrm{H}_{20} \mathrm{O}_{2}+\mathrm{Na}\right]^{+}$339.13555, found 339.13525.

3-Benzyl-8-methylchroman-4-ol (2g). The title compound was synthesized according to the general procedure employing $1 \mathrm{~g}(0.12$ mmol, $30.3 \mathrm{mg}), 1 \mathrm{~mol} \%(1.1 \mathrm{mg})$ of PC 3 , and 2.5 equiv of DIPEA $(38.8 \mathrm{mg})$. The product was purified by flash column chromatography: yield $56 \%$ (17.1 mg); anti $/$ syn $1.4: 1 ;{ }^{1} \mathrm{H}$ NMR $\left(600 \mathrm{MHz}, \mathrm{CD}_{3} \mathrm{OD}\right) \delta$ $7.30-7.21(\mathrm{~m}, 7.5 \mathrm{H}), 7.20-7.12(\mathrm{~m}, 5.5 \mathrm{H}), 7.05-7.02(\mathrm{~m}, 2.4 \mathrm{H})$, $6.99(\mathrm{~d}, J=7.3 \mathrm{~Hz}, 1.4 \times 1 \mathrm{H}$, anti), $6.80(\mathrm{t}, J=7.5 \mathrm{~Hz}, 1 \mathrm{H}$, syn), 6.73 $(\mathrm{t}, J=7.5 \mathrm{~Hz}, 1.4 \times 1 \mathrm{H}$, anti), $4.45(\mathrm{~d}, J=3.2 \mathrm{~Hz}, 1.4 \times 1 \mathrm{H}$, anti $), 4.38$ (d, $J=4.2 \mathrm{~Hz}, 1 \mathrm{H}, \operatorname{syn}$ ), 4.19 (dd, $J=11.0,2.6 \mathrm{~Hz}, 1 \mathrm{H}, s y n), 4.06-4.02$ $(\mathrm{m}, 1.4 \times 2 \mathrm{H}$, anti) $, 3.94(\mathrm{dd}, J=11.0,4.6 \mathrm{~Hz}, 1 \mathrm{H}$, syn $), 2.87(\mathrm{dd}, J=$ 13.7, $7.4 \mathrm{~Hz}, 1.4 \times 1 \mathrm{H}$, anti), $2.68(\mathrm{dd}, J=13.7,6.5 \mathrm{~Hz}, 1 \mathrm{H}$, syn), 2.58 (dd, $J=13.6,8.1 \mathrm{~Hz}, 1.4 \times 1 \mathrm{H}$, anti), $2.45(\mathrm{dd}, J=13.7,9.1 \mathrm{~Hz}, 1 \mathrm{H}$, syn $), 2.16(\mathrm{~s}, 3 \mathrm{H}$, syn $), 2.12(\mathrm{~s}, 1.4 \times 3 \mathrm{H}$, anti) $\mathrm{OH}$ signals not observed; ${ }^{13} \mathrm{C}$ NMR $\left(151 \mathrm{MHz}, \mathrm{CD}_{3} \mathrm{OD}\right) \delta 152.3,152.2,139.7,139.5$, $129.9,129.8,128.8,128.7,128.1(2 \mathrm{C}), 127.9,127.8,125.8(2 \mathrm{C}), 125.2$ (2C), 123.9, 122.7, 119.6, 119.3, 66.9, 64.7, 64.6, 64.4, 41.8, 40.6, 34.3, $32.6,14.8$ (2C); FT-IR $\nu_{\max }($ ATR $) \mathrm{cm}^{-1} 3352,2919,1598,1472$, $1258,1210,1092,1023,747,698 ; \mathrm{m} / z$ (EI) $254\left([\mathrm{M}]^{+}, 59\right), 253$ ([M $-1], 100), 91\left(\left[\mathrm{PhCH}_{2}\right]^{+}, 39\right)$; HRMS (EI) calcd for $\left[\mathrm{C}_{17} \mathrm{H}_{18} \mathrm{O}_{2}\right]^{+}$ 254.13013 , found 254.13046 .

3-Benzyl-6,8-di-tert-butylchroman-4-ol (2h). The title compound was synthesized according to the general procedure employing $\mathbf{l h}$ $(0.12 \mathrm{mmol}, 42.1 \mathrm{mg}), 1 \mathrm{~mol} \%(1.1 \mathrm{mg})$ of PC 3 , and 2.5 equiv of DIPEA $(38.8 \mathrm{mg})$. The product was purified by flash column chromatography: yield $88 \%$ (37.5 mg); anti $/$ syn $1.2: 1 ;{ }^{1} \mathrm{H}$ NMR (400 $\left.\mathrm{MHz}, \mathrm{CD}_{3} \mathrm{OD}\right) \delta 7.33-7.07(\mathrm{~m}, 15.4 \mathrm{H}), 4.48(\mathrm{~d}, J=3.5 \mathrm{~Hz}, 1 \mathrm{H}, s y n)$ $4.39(\mathrm{~d}, J=3.3 \mathrm{~Hz}, 1.2 \times 1 \mathrm{H}$, anti), $4.13(\mathrm{dd}, J=10.9,2.5 \mathrm{~Hz}, 1.2 \times$ $1 \mathrm{H}$, anti), 4.03-3.94 (m, 2H, syn), 3.90 (ddd, $J=10.9,4.0,1.1 \mathrm{~Hz}, 1.2$ $\times 1 \mathrm{H}$, anti), $2.88(\mathrm{dd}, J=13.5,7.2 \mathrm{~Hz}, 1 \mathrm{H}$, syn), $2.67(\mathrm{dd}, J=13.6,6.0$ $\mathrm{Hz}, 1.2 \times 1 \mathrm{H}$, anti), 2.57 (dd, $J=13.5,8.3 \mathrm{~Hz}, 1 \mathrm{H}$, syn), $2.42(\mathrm{dd}, J=$ 13.6, $9.8 \mathrm{~Hz}, 1.2 \times 1 \mathrm{H}$, anti), $2.23-2.15(\mathrm{~m}, 1 \mathrm{H}, \mathrm{syn}), 2.14-2.08(\mathrm{~m}$, $1.2 \times 1 \mathrm{H}$, anti), 1.37 (s, $1.2 \times 9 \mathrm{H}$, anti), 1.33 (s, 9H, syn), 1.29 (s, 1.2 $\times 9 \mathrm{H}$, anti), $1.25(\mathrm{~s}, 9 \mathrm{H}$, syn $) ;{ }^{13} \mathrm{C} \mathrm{NMR}\left(101 \mathrm{MHz}, \mathrm{CD}_{3} \mathrm{OD}\right) \delta 150.6$ (2C), 141.8, 141.5, 139.8, 139.6, 136.3, 136.1, 128.8 (2C), 128.0 (2C), $125.8,125.7,125.2,124.7,124.0,123.1,123.0,122.4,67.7,65.5,63.9$, 63.0, 41.7, 40.6, 34.5, 34.5, 34.3, 33.7, 33.7, 32.5, 30.7 (2C), 28.9 (2C); FT-IR $\nu_{\max }($ ATR $) \mathrm{cm}^{-1} 2956,2870,1479,1450,1361,1229,1171$, $1130,1028,882,749,701,532 ; \mathrm{m} / z$ (EI) $352\left([\mathrm{M}]^{+}, 65\right), 91$ $\left(\left[\mathrm{PhCH}_{2}\right]^{+}, 15\right)$; HRMS (EI) calcd for $\left[\mathrm{C}_{24} \mathrm{H}_{32} \mathrm{O}_{2}\right]^{+} 352.23968$, found 352.23972 .

3-Benzyl-8-ethoxychroman-4-ol (2i). The title compound was synthesized according to the general procedure employing $\mathbf{1 i}(0.12$ mmol, $33.8 \mathrm{mg}), 1 \mathrm{~mol} \%(1.1 \mathrm{mg})$ of PC 3 , and 2.5 equiv of DIPEA $(38.8 \mathrm{mg})$. The product was purified by flash column chromatography: yield $73 \%(25 \mathrm{mg})$; anti/syn $1: 1 ;{ }^{1} \mathrm{H}$ NMR $\left(600 \mathrm{MHz}, \mathrm{CDCl}_{3}\right) \delta$ 7.36-7.14 (m, 10H), 6.95-6.77 (m, 6H), 4.48-4.47 (m, 2H), 4.29 $(\mathrm{dd}, J=11.1,2.7 \mathrm{~Hz}, 1 \mathrm{H}), 4.22(\mathrm{ddd}, J=10.7,3.7,1.2 \mathrm{~Hz}, 1 \mathrm{H}), 4.15-$ $4.04(\mathrm{~m}, 6 \mathrm{H}), 2.88(\mathrm{dd}, J=13.7,8.4 \mathrm{~Hz}, 1 \mathrm{H}), 2.70(\mathrm{dd}, J=14.0,7.0$ 
$\mathrm{Hz}, 1 \mathrm{H}), 2.68(\mathrm{dd}, J=13.7,7.2 \mathrm{~Hz}, 1 \mathrm{H}), 2.59(\mathrm{dd}, J=13.9,8.5 \mathrm{~Hz}$, $1 \mathrm{H}), 2.35-2.29(\mathrm{~m}, 1 \mathrm{H}), 2.22(\mathrm{dqd}, J=12.9,4.3,2.8 \mathrm{~Hz}, 1 \mathrm{H}), 1.97$ (bs, $2 \mathrm{H}), 1.47-1.45(\mathrm{~m}, 6 \mathrm{H}) ;{ }^{13} \mathrm{C}$ NMR $\left(151 \mathrm{MHz}, \mathrm{CDCl}_{3}\right) \delta$ 147.6, 147.5, 144.1, 144.0, 139.2, 139.1, 129.1 (2C), 128.5 (2C), 126.3 (2C), 125.0, 124.0, 121.8, 121.6, 120.4, 120.1, 112.6 (2C), 67.4, 65.4, 65.2, 64.7, 64.4, 64.3, 41.3, 39.8, 34.6, 32.9, 14.8 (2C); FT-IR $\nu_{\max }$ (ATR) $\mathrm{cm}^{-1} 3506,2921,1585,1478,1391,1315,1255,1214,1080,1030$, 964, 900, 737; $m / z$ (EI) $284\left([\mathrm{M}]^{+}, 23\right), 91\left(\left[\mathrm{PhCH}_{2}\right]^{+}, 100\right)$; HRMS (EI) calcd for $\left[\mathrm{C}_{18} \mathrm{H}_{20} \mathrm{O}_{3}\right]^{+} 284.14070$, found 284.14077 .

3-Benzyl-6-methoxychroman-4-ol (2j). The title compound was synthesized according to the general procedure employing $\mathbf{1 j}(0.12$ mmol, $32.2 \mathrm{mg}$ ), $1 \mathrm{~mol} \%$ ( $1.1 \mathrm{mg}$ ) of PC 3 , and 2.5 equiv of DIPEA $(38.8 \mathrm{mg})$. The product was purified by flash column chromatography: yield $65 \%(21 \mathrm{mg}) ; \mathrm{mp}=112-113{ }^{\circ} \mathrm{C}$; anti $/$ syn $1: 1 ;{ }^{1} \mathrm{H}$ NMR $(400$ $\left.\mathrm{MHz}, \mathrm{CD}_{3} \mathrm{OD}\right) \delta 7.30-7.13(\mathrm{~m}, 10 \mathrm{H}), 6.88(\mathrm{~d}, J=3.0 \mathrm{~Hz}, 1 \mathrm{H})$, $6.80-6.63(\mathrm{~m}, 5 \mathrm{H}), 4.46(\mathrm{~d}, J=3.5 \mathrm{~Hz}, 1 \mathrm{H}), 4.36(\mathrm{~d}, J=4.6 \mathrm{~Hz}, 1 \mathrm{H})$, 4.09 (dd, $J=11.0,2.7 \mathrm{~Hz}, 1 \mathrm{H}), 3.99-3.89(\mathrm{~m}, 2 \mathrm{H}), 3.84$ (ddd, $J=$ 11.0, 5.1, $0.7 \mathrm{~Hz}, 1 \mathrm{H}), 3.73(\mathrm{~s}, 3 \mathrm{H}), 3.70(\mathrm{~s}, 3 \mathrm{H}), 2.87$ (dd, $J=13.6$, $7.2 \mathrm{~Hz}, 1 \mathrm{H}), 2.74(\mathrm{dd}, J=13.7,6.2 \mathrm{~Hz}, 1 \mathrm{H}), 2.56(\mathrm{dd}, J=13.6,8.3 \mathrm{~Hz}$, $1 \mathrm{H}), 2.47(\mathrm{dd}, J=13.7,9.3 \mathrm{~Hz}, 1 \mathrm{H}), 2.23-2.15(\mathrm{~m}, 1 \mathrm{H}), 2.14-2.07$ (m, $1 \mathrm{H}) ;{ }^{13} \mathrm{C}$ NMR $\left(101 \mathrm{MHz}, \mathrm{CD}_{3} \mathrm{OD}\right) \delta 153.7,153.4,148.2,148.1$, $139.7,139.5,128.8,128.7,128.0$ (2C), 125.8, 125.7, 124.9, 123.9, 116.7 (2C), 115.5 (2C), 113.9, 113.8, 67.1, 64.8, 64.5 (2C), 54.7 (2C), 42.0, 40.7, 34.3, 32.3; FT-IR $\nu_{\max }$ (ATR) $\mathrm{cm}^{-1} 2923$, 2471, 1490, 1430, $1265,1205,1161,1037,807,703 ; \mathrm{m} / z$ (EI) $270\left([\mathrm{M}]^{+}, 71\right), 91$ $\left(\left[\mathrm{PhCH}_{2}\right]^{+}, 33\right)$; HRMS (EI) calcd for $\left[\mathrm{C}_{17} \mathrm{H}_{18} \mathrm{O}_{3}\right]^{+} 270.12505$, found 270.12551 .

3-Benzyl-7-methoxychroman-4-ol (2k). ${ }^{19}$ The title compound was synthesized according to the general procedure employing $1 \mathbf{k}(0.12$ mmol, $32.2 \mathrm{mg}), 2.5 \mathrm{~mol} \%(2.74 \mathrm{mg})$ of PC 3, and 2.5 equiv of DIPEA $(38.8 \mathrm{mg})$. The product was purified by flash column chromatography: yield $92 \%(29.8 \mathrm{mg}) ; \mathrm{mp}=100-103{ }^{\circ} \mathrm{C}$; anti $/ \mathrm{syn}$ $1.7: 1 ;{ }^{1} \mathrm{H}$ NMR $\left(400 \mathrm{MHz}, \mathrm{CD}_{3} \mathrm{OD}\right) \delta 7.42-7.21(\mathrm{~m}, 7.2 \mathrm{H}), 7.20-$ $7.12(\mathrm{~m}, 8 \mathrm{H}), 7.09(\mathrm{~d}, J=8.5 \mathrm{~Hz}, 1 \mathrm{H}), 6.51(\mathrm{dd}, J=8.5,2.5 \mathrm{~Hz}, 1.7 \times$ $1 \mathrm{H}$, anti), 6.44 (dd, $J=8.5,2.5 \mathrm{~Hz}, 1 \mathrm{H}$, syn $), 6.35(\mathrm{~d}, J=2.5 \mathrm{~Hz}, 1.7 \times$ $1 \mathrm{H}$, anti), 6.30 (d, $J=2.5 \mathrm{~Hz}, 1 \mathrm{H}$, syn), $4.41(\mathrm{~d}, J=3.1 \mathrm{~Hz}, 1 \mathrm{H}, s y n)$, $4.34(\mathrm{~d}, J=3.9 \mathrm{~Hz}, 1.7 \times 1 \mathrm{H}$, anti), $4.15(\mathrm{dd}, J=11.0,2.6 \mathrm{~Hz}, 1.7 \times$ $1 \mathrm{H}$, anti), 4.11-3.93 (m, 2H, syn), 3.89 (ddd, $J=11.0,4.2,0.9 \mathrm{~Hz}, 1.7$ $\times 1 \mathrm{H}$, anti), $3.72(\mathrm{~s}, 3 \mathrm{H}$, anti $), 3.69(\mathrm{~s}, 3 \mathrm{H}, \mathrm{syn}), 2.85(\mathrm{dd}, J=13.6,7.4$ $\mathrm{Hz}, 1 \mathrm{H}$, syn), $2.66(\mathrm{dd}, J=13.7,6.6 \mathrm{~Hz}, 1.7 \times 1 \mathrm{H}$, anti), $2.57(\mathrm{dd}, J=$ 13.6, $8.0 \mathrm{~Hz}, 1 \mathrm{H}$, syn), $2.46(\mathrm{dd}, J=13.7,9.1 \mathrm{~Hz}, 1.7 \times 1 \mathrm{H}$, anti), 2.23-2.14 (m, 1H, syn), 2.14-2.03 (m, $1.7 \times 1 \mathrm{H}$, anti $) ;{ }^{13} \mathrm{C}$ NMR $\left(101 \mathrm{MHz}, \mathrm{CD}_{3} \mathrm{OD}\right) \delta 160.7$ (2C), $155.2(2 \mathrm{C}), 139.6,139.5,131.1$, $130.9,128.8,128.7,128.0$ (2C), 125.8, 125.7, 117.1, 115.6, 107.3, 106.8, 100.5 (2C), 66.3, 64.5, 64.2, 64.1, 54.3, 54.2, 42.0, 40.7, 34.2, 32.6; FT-IR $\nu_{\max }\left(\right.$ ATR) $\mathrm{cm}^{-1}: 3215,2960,1737,1667,1494,1366$, 1221, 1092, 1032, 734; $m / z(\mathrm{EI}) 270\left([\mathrm{M}]^{+}, 15\right), 91\left(\left[\mathrm{PhCH}_{2}\right]^{+}, 89\right)$; HRMS (EI) calcd for $\left[\mathrm{C}_{17} \mathrm{H}_{18} \mathrm{O}_{3}\right]^{+} 270.12505$, found 270.12534 .

3-(4-methoxybenzyl)chroman-4-ol (2l). ${ }^{20}$ The title compound was synthesized according to the general procedure employing 11 (0.12 mmol, $32.2 \mathrm{mg}), 2.5 \mathrm{~mol} \%(2.74 \mathrm{mg})$ of PC 3 , and 2.5 equiv of DIPEA $(38.8 \mathrm{mg})$. The product was purified by flash column chromatography: yield $55 \%(17.7 \mathrm{mg}) ; \mathrm{mp}=121-126{ }^{\circ} \mathrm{C}$; anti $/$ syn $1.2: 1 ;{ }^{1} \mathrm{H}$ NMR $\left(600 \mathrm{MHz}, \mathrm{CDCl}_{3}\right) \delta 7.32(\mathrm{dd}, J=7.6,1.4 \mathrm{~Hz}, 1.2 \times$ $1 \mathrm{H}$, anti), 7.25-7.17 (m, 5.4H), 7.11-7.08 (m, $1.2 \times 1 \mathrm{H}$, anti), 6.95 $(\mathrm{td}, J=7.5,0.9 \mathrm{~Hz}, 1.2 \times 1 \mathrm{H}$, anti), 6.91-6.82 (m, 7.4H), $4.50(\mathrm{t}, J=$ $3.5 \mathrm{~Hz}, 1 \mathrm{H}$, syn $), 4.48(\mathrm{t}, J=4.1 \mathrm{~Hz}, 1.2 \times 1 \mathrm{H}$, anti), $4.21(\mathrm{dd}, J=9.2$, $4.6 \mathrm{~Hz}, 1.2 \times 1 \mathrm{H}$, anti) $4.11-4.06(\mathrm{~m}, 2 \mathrm{H}, \mathrm{syn}), 3.97(\mathrm{dd}, J=11.0,4.0$ $\mathrm{Hz}, 1.2 \times 1 \mathrm{H}$, anti), $3.80(\mathrm{~s}, 3 \mathrm{H}$, syn), $3.79(\mathrm{~s}, 1.2 \times 3 \mathrm{H}$, anti), 2.82 (dd, $J=13.8,8.5 \mathrm{~Hz}, 1 \mathrm{H}$, syn), 2.68-2.58 (m, 2.2H), $2.48(\mathrm{dd}, J=$ 14.0, $9.3 \mathrm{~Hz}, 1.2 \times 1 \mathrm{H}$, anti) $, 2.32-2.24(\mathrm{~m}, 1 \mathrm{H}, \mathrm{syn}), 2.21-2.14(\mathrm{~m}$, $1.2 \times 1 \mathrm{H}$, anti), $1.91\left(\mathrm{bs}, 1.2 \times 1 \mathrm{H}\right.$, anti), $1.72(\mathrm{bs}, 1 \mathrm{H}$, syn $) ;{ }^{13} \mathrm{C} \mathrm{NMR}$ $\left(151 \mathrm{MHz}, \mathrm{CDCl}_{3}\right) \delta 158.1(2 \mathrm{C}), 154.3,154.2,131.1,131.0,130.1$ (2C), 130.0 (2C), 129.9, 129.7, 124.2, 123.2, 120.9, $120.5,116.9$ (2C), 114.0, 113.9, 67.6, 65.0, 64.9, 64.6, 55.3 (2C), 41.6, 40.1, 33.7, 31.9; FT-IR $\nu_{\max }\left(\right.$ ATR $\mathrm{cm}^{-1}: 3392,2928,1609,1582,1510,1486,1450$, $1298,1246,1176,1031,850,813,753 ; \mathrm{m} / z$ (EI) $270\left([\mathrm{M}]^{+}, 27\right), 121$ $\left(\left[4-\mathrm{MeOPhCH}_{2}\right]^{+}, 100\right)$; HRMS (EI) calcd for $\left[\mathrm{C}_{17} \mathrm{H}_{18} \mathrm{O}_{3}\right]^{+}$ 270.12505 , found 270.12548 .
3-Benzhydrylchroman-4-ol (2m). The title compound was synthesized according to the general procedure employing $1 \mathrm{~m}(0.12$ mmol, $37.7 \mathrm{mg}$ ), $1 \mathrm{~mol} \%$ ( $1.1 \mathrm{mg}$ ) of PC 3 , and 2.5 equiv of DIPEA $(38.8 \mathrm{mg})$. The product was purified by flash column chromatography: yield $86 \%$ (32.7 mg); anti/syn 1.8:1; ${ }^{1} \mathrm{H}$ NMR $\left(600 \mathrm{MHz}, \mathrm{CDCl}_{3}\right) \delta$ $7.43(\mathrm{~d}, J=7.2 \mathrm{~Hz}, 2 \mathrm{H}), 7.39(\mathrm{~d}, J=7.2 \mathrm{~Hz}, 2 \mathrm{H}), 7.35-7.18(\mathrm{~m}$, 26.7H), 7.12-7.09 (m, 3H), 6.97-6.85 (m, 5.5H), 4.44 (bs, $1 \mathrm{H}$, syn), 4.41 (bs, $1.8 \times 1 \mathrm{H}$, anti), $4.27(\mathrm{dd}, J=11.2,2.3 \mathrm{~Hz}, 1.8 \times 1 \mathrm{H}$, anti), 4.14-4.06 (m, 2H, syn), 4.00 (ddd, $J=10.8,3.5,1.5 \mathrm{~Hz}, 1 \mathrm{H}, s y n$ ), 3.96-3.93 (m, $1.8 \times 1 \mathrm{H}$, anti), $3.70(\mathrm{~d}, J=12.4 \mathrm{~Hz}, 1.8 \times 1 \mathrm{H}$, anti), 2.91 (ddd, $J=11.9,7.5,3.2 \mathrm{~Hz}, 1 \mathrm{H}$, syn), $2.79(\mathrm{dq}, J=12.4,2.3 \mathrm{~Hz}, 1.8$ $\times 1 \mathrm{H}$, anti), 2.00 (bs, $1.8 \times 1 \mathrm{H}$, anti), $1.80(\mathrm{bs}, 1 \mathrm{H}, \mathrm{syn}) ;{ }^{13} \mathrm{C} \mathrm{NMR}$ $\left(151 \mathrm{MHz}, \mathrm{CDCl}_{3}\right) \delta 154.4(2 \mathrm{C}), 142.6,142.4,142.3$ (2C), 130.9, $130.5,130.0,129.9,128.9$ (2C), 128.8 (2C), 128.1 (2C), 127.9, 127.8, $126.8,126.7,126.6,126.6,124.0,122.5,121.0,120.5,117.1,116.9$, 65.8, 64.1, 64.0, 63.1, 49.6, 49.2, 43.0, 41.9; FT-IR $\nu_{\max }($ ATR $) \mathrm{cm}^{-1}$ : 3036, 2927, 1702, 1619, 1516, 1491, 1446, 1318, 1226, 1117, 1014, 972, 755; m/z (EI) $167\left(\left[\mathrm{CHPh}_{2}\right]^{+}, 59\right), 151\left(\left[\mathrm{M}-\mathrm{CPh}_{2}\right]^{+}, 28\right)$; HRMS (EI) calcd for $\left[\mathrm{C}_{22} \mathrm{H}_{20} \mathrm{O}_{2}\right]^{+} 316.14578$, found 316.14636.

2-Benzyl-2,3-dihydro-1H-inden-1-ol (2n). The title compound was synthesized according to the general procedure employing 1 n $(0.12$ mmol, $26.7 \mathrm{mg}), 2.5 \mathrm{~mol} \%(2.74 \mathrm{mg})$ of PC 3 , and 2.5 equiv of DIPEA $(38.8 \mathrm{mg})$. The product was purified by flash column chromatography: yield $68 \%$ (14.6 mg); anti $/$ syn $2.5: 1 ;{ }^{1} \mathrm{H}$ NMR (600 $\left.\mathrm{MHz} \mathrm{CDCl}_{3}\right) \delta 7.41-7.35(\mathrm{~m}, 4 \mathrm{H}), 7.35-7.29(\mathrm{~m}, 10 \mathrm{H}), 7.28-7.21$ (m, 14.5H), 7.19-7.15 (m, 3H), $5.00(\mathrm{~d}, J=5.4 \mathrm{~Hz}, 1 \mathrm{H}$, syn), $4.95(\mathrm{~d}$, $J=6.6 \mathrm{~Hz}, 2.5 \times 1 \mathrm{H}$, anti), 3.14-3.07 (m, $4.5 \mathrm{H}), 3.01(\mathrm{dd}, J=15.3$, $7.3 \mathrm{~Hz}, 3 \mathrm{H}), 2.87-2.83(\mathrm{~m}, 2.5 \mathrm{H}), 2.81-2.75(\mathrm{~m}, 4 \mathrm{H}), 2.68(\mathrm{dq}, J=$ $14.0,7.9 \mathrm{~Hz}, 1 \mathrm{H}$, syn), 2.62-2.48 (m, 6H), 1.62 (bs, 3.5H); ${ }^{13} \mathrm{C}$ NMR $\left(151 \mathrm{MHz}, \mathrm{CDCl}_{3}\right) \delta 144.7,144.5,143.5,141.5,141.2,140.6,128.9$ (2C), 128.7, 128.5, 128.4, 128.2, 126.8 (2C), 126.2, 125.9, 125.0, 124.8, 124.7, 123.9, 80.8, 76.3, 52.3, 46.9, 39.3, 35.9, 35.8, 35.0; FT-IR $\nu_{\max }\left(\right.$ ATR $\mathrm{cm}^{-1} 3363,2932,1487,1454,1257,1102,1026,804,745$, 697; $m / z(E I) 223\left([\mathrm{M}-1]^{+}, 21\right), 132\left(\mathrm{M}-\left[\mathrm{PhCH}_{2}\right]^{+}, 100\right) 91$ $\left(\left[\mathrm{PhCH}_{2}\right]^{+}, 80\right)$; HRMS (EI) calcd for $\left[\mathrm{C}_{16} \mathrm{H}_{16} \mathrm{O}\right]^{+} 224.11957$, found 224.11989 .

3-(3-Methylbenzylidene)chroman-4-ol (2o). The title compound was synthesized according to the general procedure employing 1o $(0.12 \mathrm{mmol}, 30 \mathrm{mg}), 2.5 \mathrm{~mol} \%(2.74 \mathrm{mg})$ of PC 3 , and 2.5 equiv of DIPEA $(38.8 \mathrm{mg})$. The product was purified by flash column chromatography: yield $59 \%(17.7 \mathrm{mg}) ; \mathrm{E} / \mathrm{Z} 1: 1 ;{ }^{1} \mathrm{H}$ NMR $(400 \mathrm{MHz}$, $\left.\mathrm{CDCl}_{3}\right) \delta 7.41(\mathrm{dd}, J=7.7,1.6 \mathrm{~Hz}, 1 \mathrm{H}), 7.31-7.18(\mathrm{~m}, 6 \mathrm{H}), 7.16-$ $7.07(\mathrm{~m}, 2 \mathrm{H}), 7.06-7.00(\mathrm{~m}, 2 \mathrm{H}), 7.03-6.8(\mathrm{~m}, 6 \mathrm{H}), 6.77(\mathrm{~s}, 1 \mathrm{H})$, $5.32(\mathrm{~s}, 1 \mathrm{H}), 5.19(\mathrm{~s}, 1 \mathrm{H}), 4.97-4.86(\mathrm{~m}, 3 \mathrm{H}), 4.57(\mathrm{dd}, J=12.0,0.9$ $\mathrm{Hz}, 1 \mathrm{H}), 2.37$ (s, 3H), 2.35 (s, 3H), 2.14 (bs, $1 \mathrm{H}), 2.05(\mathrm{bs}, 1 \mathrm{H}) ;{ }^{13} \mathrm{C}$ NMR $\left(101 \mathrm{MHz}, \mathrm{CDCl}_{3}\right) \delta 154.4,154.3,138.1$ (2C), 135.5, 135.4, $134.4,133.4,131.6,130.3,130.0,129.8,129.5$ (2C), 129.2, 129.0, 128.7, 128.4, 128.3 (2C), 125.9, 125.8, 124.9, 124.0, 121.1 (2C), 117.2, $116.9,69.3,68.1,63.0,62.8,21.4(2 \mathrm{C})$; FT-IR $\nu_{\max }\left(\right.$ ATR $\mathrm{cm}^{-1} 3391$, $2922,1737,1590,1479,1370,1224,993,921,756,702 ; \mathrm{m} / z$ (EI) 252 $\left([\mathrm{M}]^{+}, 17\right), 104\left([3-\mathrm{MePh}]^{+}, 25\right), 91\left([3-\mathrm{MePhCH}]^{+}, 32\right) ; \mathrm{HRMS}$ calcd for $\left[\mathrm{C}_{17} \mathrm{H}_{16} \mathrm{O}_{2}\right]^{+} 252.11448$, found 252.11402 .

3-(4-Methoxybenzylidene)chroman-4-ol (2p). ${ }^{21}$ The title compound was synthesized according to the general procedure employing $1 \mathrm{p}(0.12 \mathrm{mmol}, 32 \mathrm{mg}), 2.5 \mathrm{~mol} \%(2.74 \mathrm{mg})$ of PC 3 , and 2.5 equiv of DIPEA $(38.8 \mathrm{mg})$. The product was purified by flash column chromatography: yield $57 \%(18.3 \mathrm{mg}), E / Z 1: 1 ;{ }^{1} \mathrm{H}$ NMR $(600 \mathrm{MHz}$, $\left.\mathrm{CDCl}_{3}\right) \delta 7.46-7.43(\mathrm{~m}, 2 \mathrm{H}), 7.42-7.39(\mathrm{~m}, 1 \mathrm{H}), 7.30-7.28(\mathrm{~m}$, $1 \mathrm{H}), 7.24-7.20(\mathrm{~m}, 2 \mathrm{H}), 7.20-7.16(\mathrm{~m}, 2 \mathrm{H}), 6.99-6.84(\mathrm{~m}, 9 \mathrm{H})$, $6.76(\mathrm{~s}, 1 \mathrm{H}), 5.33(\mathrm{~s}, 1 \mathrm{H}), 5.18(\mathrm{~s}, 1 \mathrm{H}), 4.99-4.89(\mathrm{~m}, 2 \mathrm{H}), 4.87(\mathrm{dd}$, $J=12.0,1.2 \mathrm{~Hz}, 1 \mathrm{H}), 4.59-4.55(\mathrm{~m}, 1 \mathrm{H}), 3.83(\mathrm{~s}, 3 \mathrm{H}), 3.82(\mathrm{~s}, 3 \mathrm{H})$, 2.20 (bs, $1 \mathrm{H}), 2.07$ (bs, $1 \mathrm{H}) ;{ }^{13} \mathrm{C}$ NMR $\left(151 \mathrm{MHz}, \mathrm{CDCl}_{3}\right) \delta 159.3$, $159.1,154.4,154.3,133.3,133.2,131.9,131.3,130.3,130.2,130.0$, $129.8,129.1,128.8,128.1,128.0,125.0,124.1,121.1$ (2C), 117.3, 116.9, 113.9 (2C), 69.4, 68.2, 63.0, 62.8, 55.3 (2C); FT-IR $\nu_{\max }$ (ATR) $\mathrm{cm}^{-1} 3393,2958,2929,1606,1509,1485,1458,1246,1177,1031$, 995, 828, 755; $\mathrm{m} / z$ (EI) $268\left([\mathrm{M}]^{+}, 25\right), 120\left([3-\mathrm{MeOPhCH}]^{+}, 100\right)$. 


\section{ASSOCIATED CONTENT}

\section{S Supporting Information}

The Supporting Information is available free of charge on the ACS Publications website at DOI: 10.1021/acs.joc.6b01006.

${ }^{1} \mathrm{H}$ and ${ }^{13} \mathrm{C}$ NMR spectra of products (PDF)

\section{AUTHOR INFORMATION}

\section{Corresponding Author}

*E-mail: magnus.rueping@rwth-aachen.de.

Notes

The authors declare no competing financial interest.

\section{ACKNOWLEDGMENTS}

The research leading to these results has received funding from the European Research Council under the European Union's Seventh Framework Programme (FP/2007-2013)/ERC Grant Agreement No. 617044 (SunCatChem).

\section{REFERENCES}

(1) (a) Molander, G. A.; Kenny, C. Tetrahedron Lett. 1987, 28, 4367. (b) Molander, G. A.; Kenny, C. J. Am. Chem. Soc. 1989, 111, 8236.

(2) Values calculated vs $\mathrm{Ag} / \mathrm{AgNO}_{3}$ as reference electrode in THF: (a) Shabangi, M.; Flowers, R. A., II Tetrahedron Lett. 1997, 38, 1137. (b) Flowers, R. A., II. Synlett 2008, 2008, 1427.

(3) Selected examples using stoichiometric amount of tributyltin: (a) Beckwith, A. L. J.; Roberts, D. H. J. Am. Chem. Soc. 1986, 108, 5893. (b) Ardisson, J.; Ferezou, J. P.; Julia, M.; Pancrazi, A. Tetrahedron Lett. 1987, 28, 2001. (c) Sugawara, T.; Otter, B. A.; Ueda, T. Tetrahedron Lett. 1988, 29, 75.

(4) Enholm was the first to systematically explore the scope of this reaction: (a) Enholm, E. J.; Prasad, G. Tetrahedron Lett. 1989, 30, 4939. (b) Enholm, E. J.; Burroff, J. A. Tetrahedron Lett. 1992, 33, 1835.

(5) Hays, D. S.; Fu, G. C. J. Org. Chem. 1996, 61, 4.

(6) Corey, E. J.; Pyne, S. G. Tetrahedron Lett. 1983, 24, 2821.

(7) Estévez, R. E.; Oller-López, J. L.; Robles, R.; Melgarejo, C. R.; Gansäuer, A.; Cuerva, J. M.; Oltra, J. E. Org. Lett. 2006, 8, 5433.

(8) (a) Molander, G. A.; Harris, C. R. J. Org. Chem. 1998, 63, 812. (b) Corey, E. J.; Pyne, S. G. Tetrahedron Lett. 1983, 24, 2821. (c) Streuff, J. Synthesis 2013, 45, 281.

(9) For selected reviews on photocatalysis, see: (a) Hoffmann, N. Chem. Rev. 2008, 108, 1052. (b) Balzani, V.; Credi, A.; Venturi, M. ChemSusChem 2008, 1, 26. (c) Ravelli, D.; Dondi, D.; Fagnoni, M.; Albini, A. Chem. Soc. Rev. 2009, 38, 1999. (d) Ravelli, D.; Fagnoni, M.; Albini, A. Chem. Soc. Rev. 2013, 42, 97.

(10) For selected reviews on photoredox catalysis, see: (a) Zeitler, K. Angew. Chem., Int. Ed. 2009, 48, 9785. (b) Yoon, T. P.; Ischay, M. A.; Du, J. Nat. Chem. 2010, 2, 527. (c) Narayanam, J. M. R.; Stephenson, C. R. J. Chem. Soc. Rev. 2011, 40, 102. (d) Ravelli, D.; Fagnoni, M. ChemCatChem 2012, 4, 169. (e) Prier, C. K.; Rankic, D. A.; MacMillan, D. W. C. Chem. Rev. 2013, 113, 5322.

(11) (a) Pandey, G.; Hajra, S.; Ghorai, M. K.; Kumar, K. R. J. Org. Chem. 1997, 62, 5966. (b) Tarantino, K. T.; Liu, P.; Knowles, R. R. J. Am. Chem. Soc. 2013, 135, 10022. (c) Rono, L. J.; Yayla, H. G.; Wang, D. Y.; Armstrong, M. F.; Knowles, R. R. J. Am. Chem. Soc. 2013, 135, 17735. (d) Petronijević, F. R.; Nappi, M.; MacMillan, D. W. C. J. Am. Chem. Soc. 2013, 135, 18323. (e) Griesbeck, A. G.; Reckenthäler, M. Beilstein J. Org. Chem. 2014, 10, 1143. (f) Wang, C.; Qin, J.; Shen, X.; Riedel, R.; Harms, K.; Meggers, E. Angew. Chem., Int. Ed. 2016, 55, 685.

(12) (a) Nakajima, M.; Fava, E.; Loescher, S.; Jiang, Z.; Rueping, M. Angew. Chem., Int. Ed. 2015, 54, 8828. (b) Zhu, Z.; Das, A.; Bui, L.; Zhou, H. Z.; Curran, D. P.; Rueping, M. J. Am. Chem. Soc. 2013, 135, 1823. (c) Nakajima, M.; Lefebvre, Q.; Rueping, M. Chem. Commun. 2014, 50, 3619. (d) Lefebvre, Q.; Hoffmann, N.; Rueping, M. Chem. Commun. 2016, 52, 2493.
(13) For early examples of photochemical generation of ketyl radicals and their intramolecular addition on carbon-carbon double bonds, see: (a) Belotti, D.; Cossy, J.; Pete, J.-P.; Portella, C. Tetrahedron Lett. 1985, 26, 4591. (b) Belotti, D.; Cossy, J.; Pete, J.-P.; Portella, C. J. Org. Chem. 1986, 51, 4196. For an overview for the photochemical generation of ketyl radicals and their applications, see: (c) Cossy, J.; Belotti, D. Tetrahedron 2006, 62, 6459.

(14) Ishitani, O.; Yanagida, S.; Takamuku, S.; Pac, C. J. Org. Chem. 1987, 52, 2790.

(15) For a review on PCET, see: Huynh, M. H. V.; Meyer, T. J. Chem. Rev. 2007, 107, 5004.

(16) Conti, C.; Desideri, N. Bioorg. Med. Chem. 2009, 17, 3720.

(17) Dinnocenzo, J. P.; Banach, T. E. J. Am. Chem. Soc. 1989, 111, 8646.

(18) Bentley, J.; Nilsson, P. A.; Parsons, A. F. J. Chem. Soc. Perkin Trans. 1 2002, 12, 1461.

(19) Sangwan, N. K.; Rastogi, S. N. Indian J. Chem. B 1980, 19, 500.

(20) Gomis, M.; Kirkiacharian, B. S.; Likforman, J.; Mahuteau, J. Bull. Soc. Chim. Fr. 1988, 3, 585.

(21) Pivnenko, N. S.; Orlov, V. D.; Nodel'man, O. A.; Mikhed'kina, E. I.; Lavrushin, V. F. J. Org. Chem. USSR (Engl. Trans.) 1980, 16, 1657. 